\title{
\begin{tabular}{lllllllllllll}
$\mathrm{A}$ & $\mathrm{R}$ & $\mathrm{T}$ & $\mathrm{I}$ & $\mathrm{C}$ & $\mathrm{U}$ & $\mathrm{L}$ & $\mathrm{O}$ \\
\hline
\end{tabular} \\ Estructura de bicapas Mo/CulnSe para células solares de lámina delgada
}

\author{
C. GUILLÉN, J. HERRERO \\ Departamento de Energías Renovables (CIEMAT). Avda. Complutense 22, Madrid-28040.
}

\begin{abstract}
La optimización de las características estructurales y morfológicas del sistema formado por películas de molibdeno y seleniuro de cobre e indio depositadas sobre sustratos de vidrio (vidrio/Mo/CuInSe ${ }_{2}$ ) resulta esencial para su aplicación en la formación de células solares de lámina delgada eficientes. Para el desarrollo de este tipo de sistemas se han utilizado sustratos de vidrio sodocálcico, sobre los que se han preparado láminas delgadas de Mo mediante evaporación a ritmos de crecimiento de entre 4 y 13 A / s, y a continuación películas de CuInSe ${ }_{2}$ mediante electrodepósito directo a una velocidad constante de $12 \AA / \mathrm{s}$. El análisis estructural y morfológico de las muestras preparadas indica que la rugosidad superficial de la película de $\mathrm{CuInSe}_{2}$ se minimiza, y su tamaño de grano se maximiza, cuando el electrodepósito se realiza sobre láminas delgadas de Mo evaporadas a velocidades superiores a $8 \AA / \mathrm{s}$.
\end{abstract}

Palabras clave: láminas delgadas, evaporación, electrodepósito, estructura, morfología.

\section{Structure of $\mathrm{Mo} / \mathrm{CuInSe}{ }_{2}$ bilayers for thin film solar cells}

The optimization of the structural and morphological characteristics of the system constituted by molibdenum and copper indium diselenide layers deposited onto glass substrates (glass $/ \mathrm{Mo} / \mathrm{CuInSe}_{2}$ ) is essential for its application in efficient thin film solar cells. For developing such kind of systems, soda-lime glass substrates have been used to prepare Mo thin films by evaporation at deposition rates between 4 and $13 \AA$ A , and subsequently $\mathrm{CuInSe} \mathrm{e}_{2}$ layers by direct electrodeposition at $12 \AA / \mathrm{s}$ constant rate. The structural and morphological analysis of the samples indicated that the surface roughness of the $\mathrm{CuInSe}_{2}$ layer is minimized, and its grain size is maximized, when electrodeposition process takes place onto Mo thin films evaporated at rates higher than $8 \AA / \mathrm{s}$.

Key words: Thin films, evaporation, electrodeposition, structure, morphology.

\section{INTRODUCCIÓN}

El análisis estructural y morfológico del sistema formado por películas de molibdeno y seleniuro de cobre e indio depositadas sobre vidrio, heteroestructuras de la forma vidrio/Mo/CuInSe $2^{\prime}$ se han mostrado de gran importancia para el desarrollo de células solares de lámina delgada de alta eficiencia $(1,2)$. En particular, resulta necesario maximizar el tamaño de grano y minimizar la rugosidad superficial de la película de $\mathrm{CuInSe}_{2}$ para hacer mínima la densidad de defectos y mejorar la calidad final de la célula solar. En el caso de los sistemas formados por materiales policristalinos en lámina delgada, las características estructurales y morfológicas están relacionadas con la tensión de la red cristalina en cada uno de los componentes, debido a la influencia sobre las propiedades de los materiales de los defectos inducidos durante los procesos de tensión y relajación que tienen lugar durante el crecimiento o tratamiento posterior de dichos componentes.

Las propiedades mecánicas de películas delgadas sobre sustratos son diferentes de las de los materiales en volumen, debido a sus características especiales de microestructura y densidad, la gran proporción de superficie respecto del volumen, las dimensiones reducidas y el confinamiento impuestos por el sustrato. Generalmente las propiedades resultantes del recubrimiento están fuertemente influenciadas por el método de depósito y las condiciones de preparación. En el caso de láminas delgadas de molibdeno depositadas mediante pulverización catódica o evaporación sobre sustratos de vidrio, sus propiedades eléctricas, estructurales y morfológicas se han encontrado fuertemente dependientes de los parámetros de preparación $(3,4)$. Asimismo se ha observado que las características de los sustratos utilizados afectan a las propiedades estructurales de las películas de $\mathrm{CuInSe}_{2}$ evaporadas sobre ellos (2).
En el presente trabajo se han desarrollado heteroestructuras de la forma vidrio/Mo/CuInSe ${ }_{2}$, depositando sobre sustratos de vidrio láminas delgadas de Mo mediante evaporación por cañón de electrones, y utilizando dichos vidrios recubiertos con molibdeno como electrodos para la preparación de películas de $\mathrm{CuInSe}_{2}$ mediante electrodepósito directo. Se han realizado análisis estructural y morfológico de las muestras desarrolladas, para determinar la influencia de las características del recubrimiento de Mo sobre la tensión estructural, el tamaño de grano y la rugosidad superficial de las láminas de CuInSe ${ }_{2}$ electrodepositadas.

\section{PARTE EXPERIMENTAL}

Sobre sustratos de vidrio sodocálcico de $2 \mathrm{~mm}$ de espesor se prepararon láminas delgadas de molibdeno de aproximadamente $1 \mu \mathrm{m}$ de espesor mediante evaporación producida por cañón de electrones. La evaporación se llevó a cabo en el sistema experimental descrito en

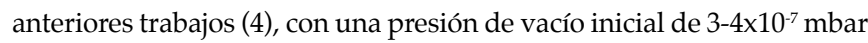
y presiones máximas de $7-8 \times 10^{-7}$ mbar durante el proceso. Las características estructurales de la lámina de Mo resultan dependientes de la velocidad de crecimiento, que se ha mantenido constante con valores entre 4 y $13 \AA$ As. A continuación, se prepararon películas de CuInSe ${ }_{2}$ mediante electrodepósito directo sobre los vidrios cubiertos con Mo evaporado. El proceso de electrodepósito se realizó a partir de una disolución acuosa conteniendo sulfatos de cobre y de indio, con ácido de selenio (5), ajustando el potencial y el tiempo de depósito para obtener láminas de composición estequiométrica de $2 \mu \mathrm{m}$ de espesor, con un 
ritmo de crecimiento constante de aproximadamente $12 \AA / \mathrm{s}$. Todos los procesos de preparación se realizaron a temperatura ambiente.

Las muestras preparadas se han analizado mediante la técnica de difracción de rayos X (XRD) en la geometría Bragg-Brentano, que ha permitido determinar la orientación cristalográfica preferencial y la tensión estructural en la dirección de crecimiento (perpendicular a la superficie) de las láminas delgadas de $\mathrm{CuInSe}_{2}$ electrodepositadas sobre Mo evaporado. La morfología y el tamaño de grano de las muestras han sido observados mediante microscopía de barrido electrónico (SEM). El valor medio de la rugosidad superficial de las láminas se ha determinado mediante un perfilómetro.

\section{RESULTADOS Y DISCUSIÓN}

En los diagramas de difracción de rayos $\mathrm{X}$ que se muestran en la figura 1, obtenidos para las muestras de vidrio/ $\mathrm{Mo} / \mathrm{CuInSe}_{2}$ desarrolladas, se identificaron como picos de difracción principales los correspondientes a los planos cristalográficos $\langle 110\rangle$ del Mo de estructura cúbica (6) y a los planos $<112>$ del CuInSe ${ }_{2}$ de estructura tetragonal (7). La posición angular y la anchura de estos picos de difracción principales se han encontrado dependientes de la velocidad de crecimiento de la película de molibdeno. En la figura 2 puede observarse que al aumentar dicha velocidad de crecimiento disminuye el espaciado interplanar y aumenta la anchura del pico de difracción $<110>$ del Mo. Por otra parte, en la figura 3 se observa que el espaciado interplanar y la anchura del pico $<112>$ del CuInSe, se ven a su vez afectados por las características de la película de Mo utilizada como sustrato.

Los datos obtenidos para el espaciado interplanar $<\mathrm{hkl}>$ están relacionados con la tensión estructural del material en lámina delgada por medio de la expresión: $\varepsilon_{\mathrm{hkl}}=\left(\mathrm{d}_{\mathrm{hkl}}-\mathrm{d}_{0}\right) / \mathrm{d}_{0}$, donde $\mathrm{d}_{0}$ es igual al espaciado interplanar del material en equilibrio, que es de $2.225 \AA$ para los planos $<110>$ del Mo (6), y $3.34 \AA$ A para los planos $<112>$ del $\mathrm{CuInSe}_{2}$ (7). Comparando dichos valores con los representados en las figuras 2 y 3 , observamos que las láminas de Mo evaporadas presentan tensiones estructurales de signo negativo $\left(\varepsilon_{110}<0\right)$, denominadas com-

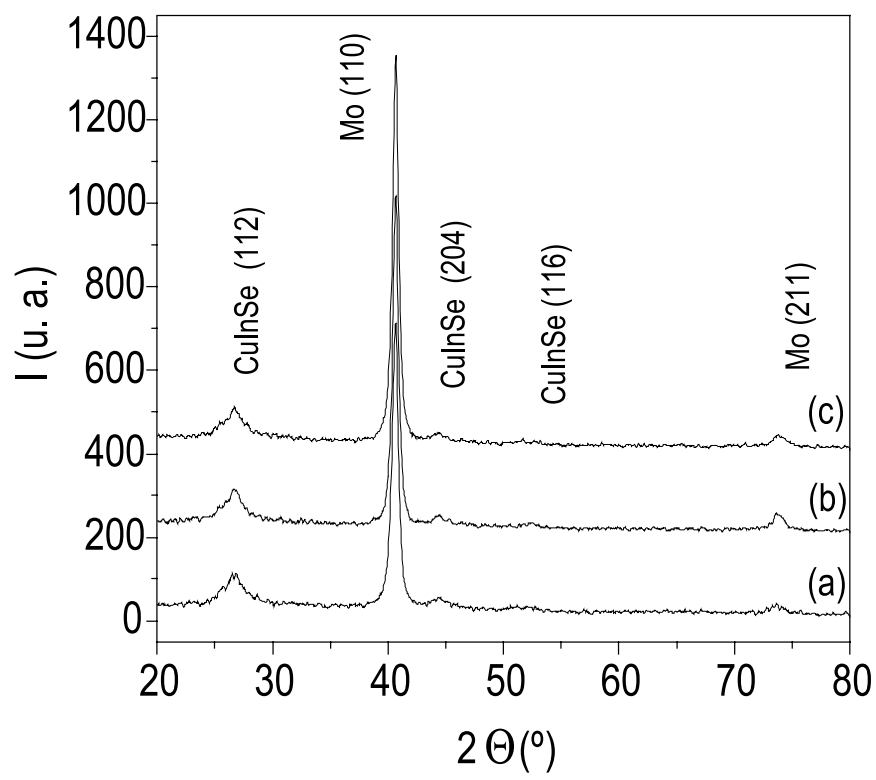

Figura 1: Diagramas de difracción de rayos $X$ correspondientes a láminas de CuInSe $e_{2}$ electrodepositadas sobre películas de Mo evaporadas a distintas velocidades: (a) $\mathrm{r}_{\mathrm{Mo}}=4 \AA / \mathrm{s}$, (b) $\mathrm{r}_{\mathrm{Mo}}=8 \AA / \mathrm{s} \mathrm{y} \mathrm{(c)} \mathrm{r}_{\mathrm{Mo}}=$ $13 \AA / s$. presivas, mientras que las películas de $\mathrm{CuInSe}_{2}$ electrodepositadas presentan tensiones de signo positivo $\left(\varepsilon_{112}>0\right)$, denominadas extensivas o tensiles. La tensión estructural en las láminas de Mo aumenta con la velocidad de crecimiento, lo que se ha relacionado con el aumento en la densidad o compacidad de la lámina $(3,4)$. En cuanto a los valores de tensión obtenidos en las láminas delgadas de $\mathrm{CuInSe}_{2}$ electrodepositadas sobre Mo, están relacionados (8) con el factor de desajuste de red $\mathrm{f}_{\mathrm{m}}=\left(\mathrm{a}_{\text {CulnSe2 }}-\mathrm{a}_{\mathrm{Mo}}\right) / \mathrm{a}_{\mathrm{Mo}}$, donde $\mathrm{a}_{\text {CuInSe2 }} \approx(1 / 2) \mathrm{c}_{\text {CuInSe2 }}$ es el parámetro de red del CuInSe ${ }_{2}$ que en equilibrio es $5.782 \AA(7), \mathrm{y} \mathrm{a}_{\mathrm{Mo}}$ es el parámetro de red del Mo, en equilibrio de $3.147 \AA$ (6). La diferencia positiva entre estos valores es responsable de la existencia de una tensión extensiva o tensil en la lámina de $\mathrm{CuInSe}_{2^{\prime}}$ esta tensión aumenta cuando disminuye el parámetro de red de la película de Mo que se utiliza como sustrato. Por otra parte, la influencia del tamaño de grano en la tensión que presentan las láminas delgadas policristalinas ha sido recientemente analizada por diferentes autores (9), que han indicado la existencia de tensiones extensivas en las regiones próximas a las fronteras de grano, que disminuyen cuando aumenta el radio del grano. Las imágenes obtenidas mediante microscopio de barrido electrónico, tales como las

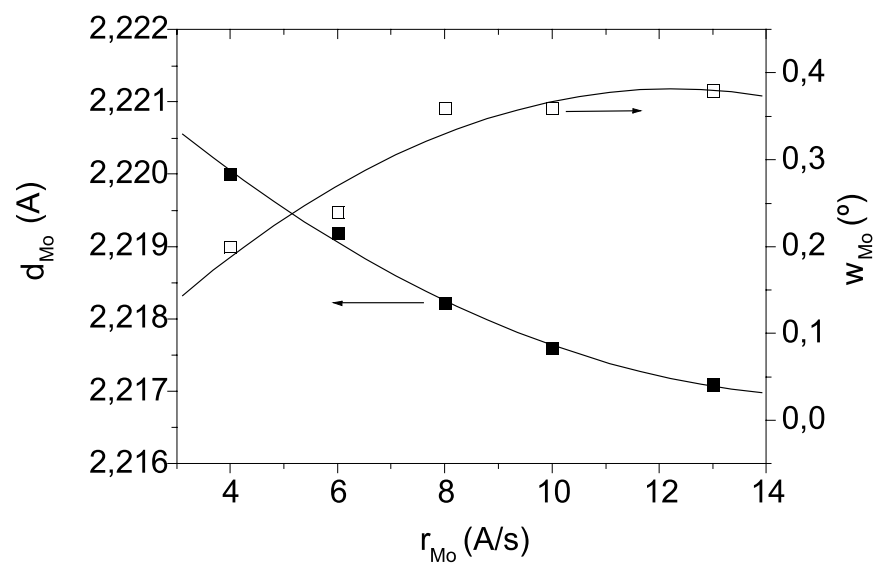

Figura 2: Espaciado interplanar y anchura del pico de difracción $<110>$ del Mo en función de la velocidad de crecimiento de la lámina.

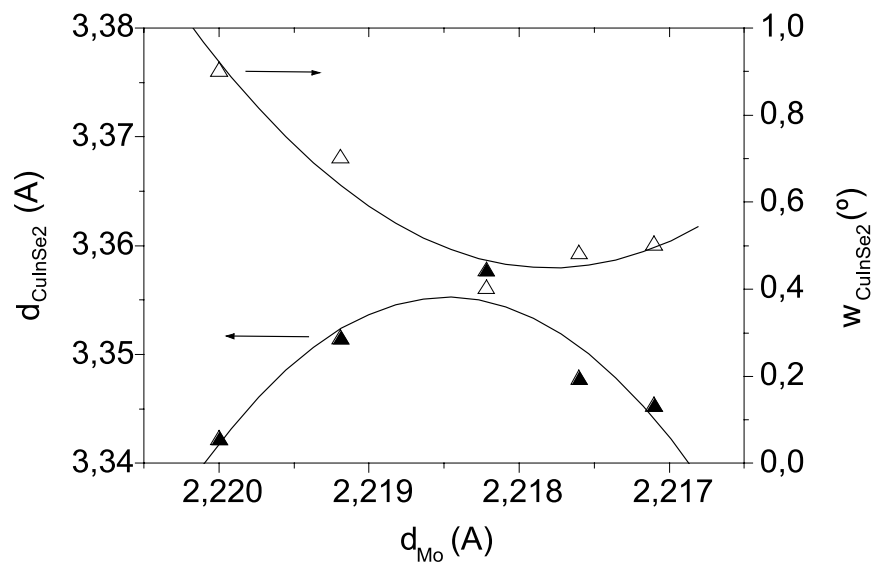

Figura 3: Espaciado interplanar y anchura del pico de difracción $<112>$ del CuInSe ${ }_{2}$ en función de la distancia interplanar en el sustrato de Mo. 
(a)

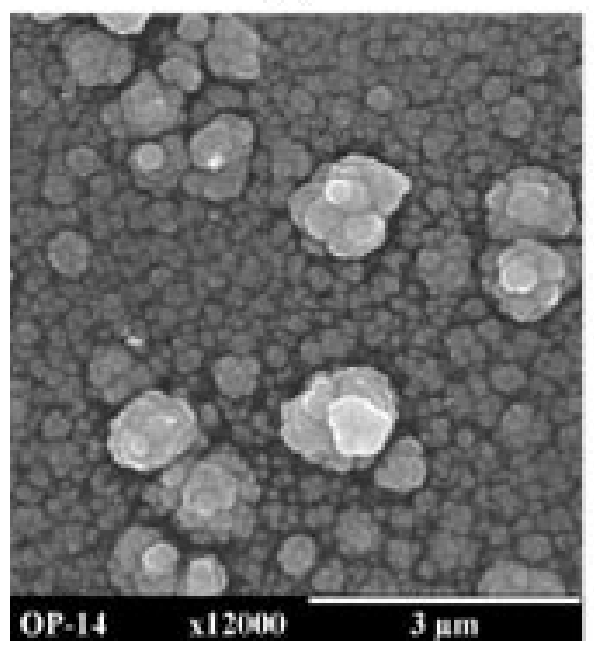

(b)

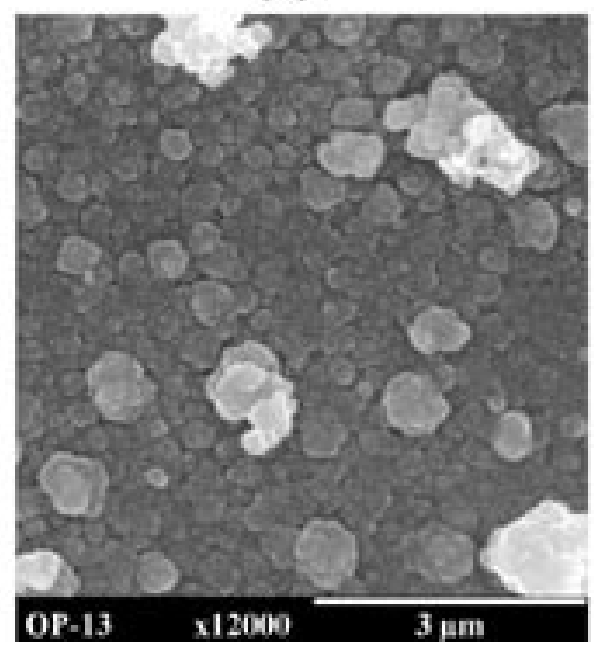

(c)

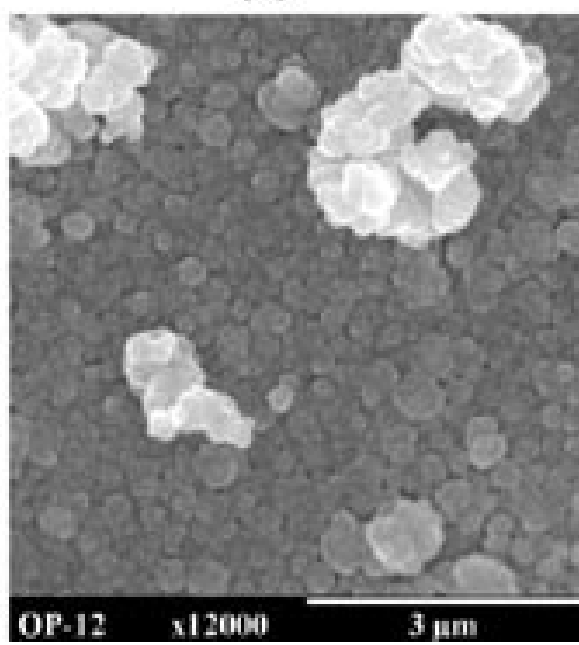

Figura 4: Imágenes de microscopio electrónico para láminas de CuInSe ${ }_{2}$ electrodepositadas sobre películas de Mo evaporadas a distintas velocidades: (a) $\mathrm{r}_{\mathrm{Mo}}=4 \AA / \mathrm{s}$, (b) $\mathrm{r}_{\mathrm{Mo}}=8 \AA / \mathrm{s}$ y (c) $\mathrm{r}_{\mathrm{Mo}}=13 \AA / \mathrm{s}$.

que se ilustran en la figura 4, indican que el tamaño de grano de las láminas de CuInSe $e_{2}$ electrodepositadas aumenta al aumentar la velocidad de crecimiento de la película de Mo utilizada como sustrato. Así pues, al aumentar el tamaño de grano, disminuye la tensión extensiva asociada para estas láminas, tal como se había observado en la figura 3. Las láminas de $\mathrm{CuInSe}_{2}$ con mayores tamaños de grano presentaron también una menor rugosidad superficial, tal como puede verse en la figura 5, con lo que sus características morfológicas pueden considerarse óptimas.

\section{CONCLUSIONES}

Mediante la evaporación de láminas delgadas de molibdeno sobre sustratos de vidrio, y el posterior electrodepósito de películas de seleniuro de cobre e indio, se han desarrollado estructuras de la forma vidrio/Mo/CuInSe $e_{2}$ que presentan tensiones estructurales de tipo compresivo en la lámina de Mo y de tipo extensivo en la de CuInSe ${ }_{2}$ aumentando en ambas los valores de tensión al aumentar la velocidad de crecimiento de la lámina de Mo. El aumento de la tensión estructural extensiva en la lámina de CuInSe ${ }_{2}$ favorece el aumento del tamaño de grano y la disminución de la rugosidad superficial, por lo que es posible optimizar las características morfológicas de las películas de $\mathrm{CuInSe}_{2}$ electrodepositadas utilizando como sustratos láminas de Mo evaporadas a velocidades superiores a $8 \AA$ /s.

\section{AGRADECIMIENTOS}

El presente trabajo se ha realizado dentro de los proyectos "Desarrollo de materiales y dispositivos fotovoltaicos" financiado por el CIEMAT, y "MARISOL" financiado por la Comunidad de Madrid.

\section{REFERENCIAS}

1. M. A. Contreras, B. Egaas, K. Ramanathan, J. Hiltner, A. Swartzlander, F. Hasoon y R. Noufi, Progress in Photovoltaics 7 (1999) 311-316.

2. M. A. Contreras, K. M. Jones, L. Gedvilas y R. Matson, Actas de la " $16^{\text {th }}$ European Photovoltaic Solar Energy Conference", Glasgow (2000), pp. 732-735.

3. J. H. Scofield, A. Duda, D. Albin, B. L. Ballard y P. K. Predecki, Thin Solid Films 260 (1995) 26.

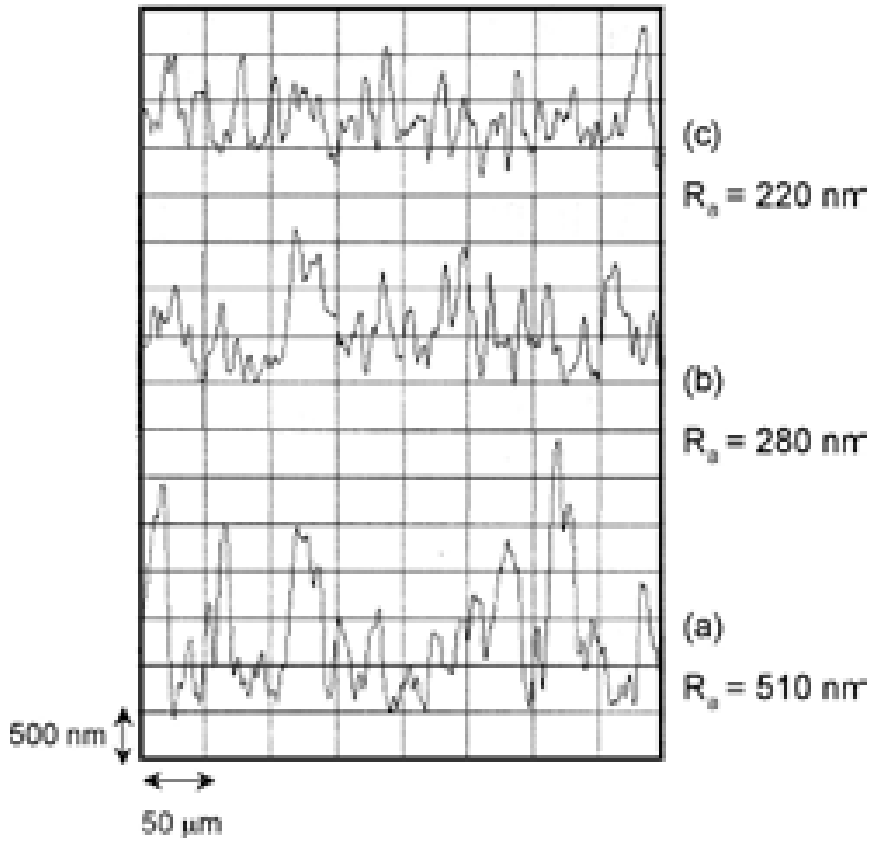

Figura 5: Rugosidad superficial de las láminas de CuInSe ${ }_{2}$ electrodepositadas sobre películas de Mo evaporadas a distintas velocidades: (a) $\mathrm{r}_{\mathrm{Mo}}=4 \AA / \mathrm{s}$, (b) $\mathrm{r}_{\mathrm{Mo}}=8 \AA / \mathrm{s} \mathrm{y} \mathrm{(c)} \mathrm{r}_{\mathrm{Mo}}=13 \AA / \mathrm{s}$.

4. C. Guillén y J. Herrero, Actas de la "International Conference on Advanced Materials and Processing Technologies", Madrid (2001), pp. 575-581.

5. C. Guillén y J. Herrero, J. Electrochemical Society 142 (1995) 1834-1838.

6. Ficha de difracción del Mo, $\mathrm{n}^{\circ}$ 4-809 según el "Joint Committee on Powder Diffraction Standards".

7. Ficha de difracción del CuInSe ${ }_{2^{\prime}} \mathrm{n}^{\circ}$ 23-209 según el "Joint Committee on Powder Diffraction Standards".

8. H. Metzner, Th. Hahn, J. Cieslak, J. Eberhardt, M. Müller, U. Reislöhner, U. Kaiser, A. Chuvilin, J. Kräusslich y W. Witthuhn, J. Phys. Chem. Solids 64 (2003) 1491-1494.

9. R. Castro-Rodríguez, V. Sosa, A. I. Oliva, A. Iribarren, J. L. Peña y F. Caballero-Briones, Thin Solid Films 373 (2000) 6-9.

Recibido: 1.2 .03

Aceptado: 30.11 .03 